\title{
Capsule Commentary on Ott et al., Do Statins Impair Cognition? A Systematic Review and Meta-analysis of Randomized Controlled Trials
}

\author{
Huseyin Naci, Ph.D \\ LSE Health, London School of Economics and Political Science, London, UK.
}

J Gen Intern Med 30(3):347

DOI: $10.1007 / \mathrm{s} 11606-014-3158-5$

(๑) Society of General Internal Medicine 2015

$I^{n}$ $\mathrm{n}$ this issue of the Journal, Ott and colleagues report the findings of their systematic review and meta-analysis, which evaluates whether the use of statins is responsible for cognition impairment in randomized controlled trials. ${ }^{1}$ This is the most comprehensive review to date evaluating the causal relationship between statin use and cognitive impairment. The authors did a commendable job in compiling a comprehensive list of relevant randomized controlled trials, performing extensive statistical analyses, and interpreting their findings with careful clinical insight.

The authors' laudably cautious interpretation of their findings confirms that existing randomized controlled trial evidence does not support the US Food and Drug Administration (FDA) warning about potential adverse effects of statins on cognition. This is an important, timely, and clinically meaningful finding: clinicians, patients, and their caregivers can take comfort in knowing that there does not appear to be a causal relationship between statin use and cognitive impairment.

Beyond its clinical implications, this study has two important lessons for drug regulators. First, there is discordance between the results of randomized controlled trials and reports from the Adverse Events Reporting System. Such discordance is unsurprising. Previous research has shown that findings of randomized and non-randomized study designs may differ, and the magnitude and direction of this difference cannot be systematically estimated. A recent study showed that even high-quality observational analyses informing FDA decisions on adverse events produce findings that contradict those found in randomized controlled trials. ${ }^{2}$

Second, FDA warnings on drug-related adverse events can have unintended consequences. In a recent study, the FDA's safety warnings about antidepressants were shown to significantly decrease antidepressant use-well beyond levels that the regulators had intended. ${ }^{3}$ Although a similar evaluation is lacking for FDA's warning on statins, it is possible that individuals stopped taking their pills or took smaller doses as an unintended result of the widespread media coverage that publicized the FDA's decision on statins.

Regulatory decisions on medications should be based on current best evidence: up-to-date information from relevant, valid research. Although previous reviews on this topic called for additional larger, better designed studies, ${ }^{4}$ Ott and colleagues settle the question about the cognitive harms of statins.

Conflict of interest: The author has no conflicts of interest with this article.

Corresponding Author: Huseyin Naci, Ph.D; LSE Health, London School of Economics and Political Science, London, UK (e-mail: h.naci@lse.ac.uk).

\section{REFERENCES}

1. Ott, BR, Daiello LA, Dahabreh IJ, Springate BA, Bixby K, Murali M, Trikalinos TA. Do statins impair cognition? A systematic review and metaanalysis of randomized controlled trials. J Gen Intern Med. doi:10.1007/ s11606-014-3115-3.

2. Sipahi I, Celik S, Tozun N. A comparison of results of the US food and drug administration's mini-sentinel program with randomized clinical trials: the case of gastrointestinal tract bleeding with dabigatran. JAMA Intern Med. 2014; 174(1):150-1.

3. Lu Christine Y, Zhang F, Lakoma MD, Madden JM, Rusinak D, Penfold RB, et al. Changes in antidepressant use by young people and suicidal behavior after FDA warnings and media coverage: quasi-experimental study. BMJ. 2014;348:g3596.

4. Richardson K, Schoen M, French B, Umscheid CA, Mitchell MD, Arnold SE, et al. Statins and cognitive function: a systematic review. Ann Intern Med. 2013;159:688-97. doi:10.7326/0003-4819-159-10201311190-00007. 